\title{
Organocatalytic Silicon-Free SuFEx reactions for modular synthesis of sulfonates and sulfonamides
}

\author{
Muze Lin ${ }^{1}$, Jinyun $\mathrm{Luo}^{1}, \mathrm{Yu} \mathrm{Xie}^{1}$, Guangfen $\mathrm{Du}^{1 *}$, Zhihua Cai ${ }^{1}$, Bin Dai ${ }^{1 *}$ and $\mathrm{Lin} \mathrm{He}^{1^{*}}$
}

\begin{abstract}
Sulfur(VI) fluoride exchange (SuFEx) click chemistry provides a powerful tool for rapid construction of modular connections. Here, we report a novel catalytic silicon-free SuFEx reaction with sulfonyl fluorides. Under the catalysis of $10 \mathrm{~mol} \%$ N-heterocyclic carbene (NHC), a range of phenols and alcohols react with different sulfonyl fluorides to afford sulfonate esters in $49-99 \%$ yields. In addition, Under the relay catalysis of $10 \quad \mathrm{~mol} \%$ N-heterocyclic carbene and $10 \quad \mathrm{~mol} \%$
\end{abstract} 1-hydroxybenzotriazole (HOBt), a variety of primary and secondary amines react with different sulfonyl fluorides to produce sulfonamides in 58\%-99\% yields. More than 140 sulfonylated products, including 17 natural product derivatives have been prepared through this method. Mechanism study showed that NHCs might act as a carbon-centered Brønsted base to catalyse the SuFEx click reactions via the formation of hydrogen bonding with phenols or alcohols.

Sulfonats and sulfonamides are key structural motifs that exist widely in numerous pharmaceuticals, biologically active molecules and advanced functional materials ${ }^{1-3}$. Owing to their unique physicochemical properties, a variety of sulfonate- and sulfonamide-containing drugs have been developed and used widely for antibacterial, anti-tumoral, anti-inflammatory and other treatments ${ }^{4-6}$. Accordingly, tremendous eff-

${ }^{1}$ Key Laboratory for Green Processing of Chemical Engineering of Xinjiang Bingtuan/ School of Chemistry and Chemical Engineering, Shihezi University Xinjiang Uygur Autonomous Region, 832000, People's Republic of China._E-mail: duguangfen@shzu.edu.cn; db_tea@shzu.edu.cn, helin@shzu.edu.cn. 
orts have been devoted to the development of new methods for the construction of these valuable sulfonyl-containing compounds ${ }^{7-11}$. To date, the esterification reaction of sulfonyl chlorides with alcohols and the amination reaction of sulfonyl chlorides with amines provide the most straightforward and general method for the synthesis of sulfonates and sulfonamides, respectively. However, these traditional methods encounter some issues ${ }^{12}$, such as the inherent instability and high moisture sensitivity of sulfonyl chlorides, the competing chlorination reaction, and the facile reduction of $\mathrm{S}^{\mathrm{VI}}$ to $\mathrm{S}^{\mathrm{IV}}$. Therefore, the development of novel and robust protocol for the construction of these sulfonated compounds is highly significant. Different to sulfonyl chlorides, sulfonyl fluorides exhibit remarkable thermodynamic and redox stability. Based on these unique properties of $\mathrm{S}^{\mathrm{VI}}-\mathrm{F}$ bond, Sharpless and coworkers developed sulfur(VI)-fluoride exchange click chemistry ${ }^{13-15}$ in 2014. This new generation click chemistry provides powerful technology for rapid assembly of functional molecules through $\mathrm{S}^{\mathrm{VI}}$-F exchange with different nucleophiles, which has been employed widely in organic synthesis ${ }^{16-21}$, chemical biology $y^{22-25}$, drug discovery ${ }^{26-28}$ and polymer chemistry $^{29-31}$. SuFEx click reactions of sulfonyl fluorides provide an attractive alternative approach for the synthesis of sulfonates and sulfonamides. However, the reduced reactivity of sulfonyl fluorides is a great challenge for the reaction. Although base-catalysed SuFEx reactions of sulfonyl fluorides and silyl ethers have been well established $^{13,17,18,29-31}$, the silicon-free reaction of sulfonyl fluorides is still a challenge for the research. Very recently, Moses $^{32}$ and coworkers introduced a very interesting accelerated SuFEx click reaction. They found that under the catalysis of 1-20 mol\% 
Barton's base, various aryl and alkyl alcohols react with different SuFExable hubs efficiently to afford a range of products. However, authors found that stoichiometric amount of silicon additive hexamethyldisilazane was required for the reaction, which was assumed to react with alcohol and led to the in situ generation of the reactive silyl ether intermediates. On the other hand, compared to the extensively studied SuFEx click reactions of sulfonyl fluorides and silyl ethers, the analogous click reactions of amines are underdeveloped. In 2018, Ball, am Ende and coworkers reported ${ }^{33}$ an $\mathrm{Ca}\left(\mathrm{NTf}_{2}\right)_{2}$ mediated SuFEx reaction of sulfonyl fluorides and amines for the synthesis of sulfonamides. However, the reaction was not catalytic and stoichiometric amount of $\mathrm{Ca}\left(\mathrm{NTf}_{2}\right)_{2}$ was required in order to get good reaction yields. Very recently, Li and coworkers $^{34}$ documented an interesting HOBt-catalysed SuFEx reaction of sulfonyl fluorides with amines, which offers a novel methodology for facile access to sterically hindered sulfonamides. However, the authors revealed that excess of silicon additive TMDS and organic base were required for the reaction. Despite significant progress made in this research field, general catalytic silicon- and base-free methods for SuFEx click reactions of sulfonyl fluorides are still unavailable.

As an important type of organocatalyst, N-heterocyclic carbenes (NHCs) have been used widely in organic synthesis ${ }^{35-37}$. Based on the strong Lewis basicity of NHCs, a broad variety of transformations, including benzoin reaction ${ }^{38-39}$, Stetter reaction ${ }^{40-41}$, homoenolate transformations ${ }^{42-45}$, redox reactions ${ }^{46-47}$, photo-reactions ${ }^{48-50}$ and other reactions $s^{51-54}$ have been intensively studied. In sharp contrast, reactions based on the Brønsted basic properties of NHCs are far less examined. To date, only several very 
limited reactions, such as NHC-catalysed transesterifications ${ }^{55-56}$ and Michael additions ${ }^{57-60}$ had been reported. In these reactions, NHCs acted as strong Brønsted base to activate proton-containing nucleophiles through the formation of hydrogen bonding. We reasoned that NHCs could also be employed as Brønsted base to catalyse the SuFEx click reactions of sulfonyl fluorides with alcohols and amines to construct sulfonates and sulfonamides. Herein, we would like to report this result (Fig. 1c).

\section{Results}

Optimization studies. As shown in Table 1, our study commenced by selecting commercially available sulfonyl fluoride $\mathbf{1 a}$ and phenol 2a as the model substrates for optimization of the reaction conditions. To our delight, in the presence of $4 \AA$ molecular sieves and $10 \mathrm{~mol} \%$ of a stable NHC, A ((1,3-bis(2,6-dissopropylphenyl)imidazole-2-ylidene, IPr), the reaction proceeded smoothly in acetonitrile at ambient temperature to afford the desired sulfonic ester $\mathbf{3 a}$ in $97 \%$ yield (Table 1, entry 1). Encouraged by this success, several other common NHCs were then evaluated. NHCs generated from imidazolium B1, B2, B3 and imidazolinium $\mathbf{C}$ catalysed the reaction efficiently to give 3a in excellent yield (Table 1, entries 2-5). Thiazolium D derived NHC showed moderate catalytic reactivity for the reaction (Table 1, entry 6). NHC derived from triazolium E1 catalysed the reaction in $94 \%$ yield, whereas perfluorophenyl substituted triazolium E2 catalysed the reaction in very low yield owing to the lower basicity (Table 1, entries 7 and 8). A brief screening of the reaction media showed that toluene, dichloromethane and THF are all suitable for the reaction, giving the desired product in excellent yields, whereas dichloroethane and 
1,4-dioxane showed low efficiency (Table 1, entries 9-13). Reduction of NHC loading to $5 \mathrm{~mol} \%$ led to slightly decreased reaction yield (Table 1, entry 14). However, further reducing the catalyst loading to $2 \mathrm{~mol} \%$ resulted in dramatic decrease of reaction efficiency (Table 1, entry 15). The excellent yield of 3a was maintained when the loading of sulfonyl fluoride 1a was reduced to 1.2 equiv. (Table 1, entry 16).

\section{Table 1 Optimization of reaction conditions ${ }^{\text {a }}$}<smiles>Cc1ccc(S(=O)(=O)F)cc1</smiles>

1a<smiles>Oc1ccccc1</smiles>

2a

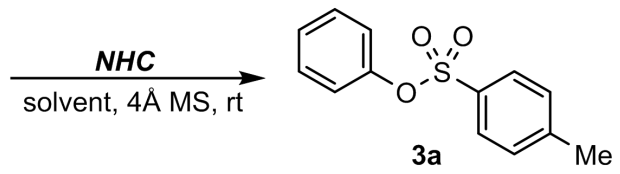

3a
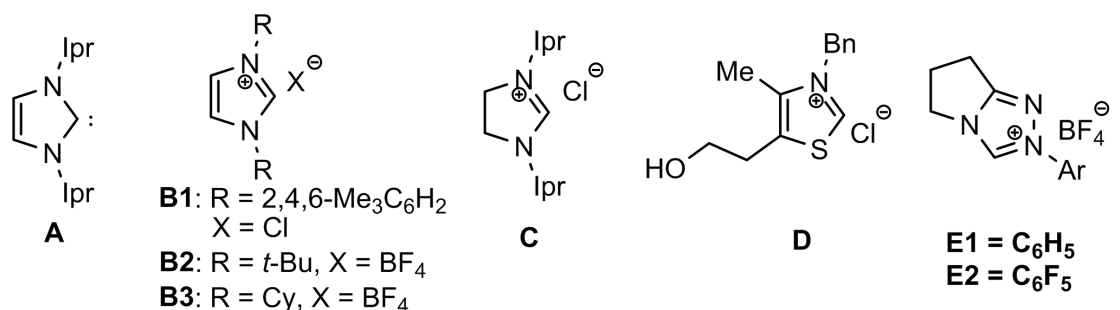

\begin{tabular}{|c|c|c|c|c|}
\hline Entry & Catalyst & Solvent & Time (h) & Yield $(\%)^{[b]}$ \\
\hline 1 & $\mathbf{A}$ & $\mathrm{CH}_{3} \mathrm{CN}$ & 24 & 97 \\
\hline 2 & B1 & $\mathrm{CH}_{3} \mathrm{CN}$ & 24 & 99 \\
\hline 3 & B2 & $\mathrm{CH}_{3} \mathrm{CN}$ & 24 & 96 \\
\hline 4 & B3 & $\mathrm{CH}_{3} \mathrm{CN}$ & 24 & 98 \\
\hline 5 & $\mathbf{C}$ & $\mathrm{CH}_{3} \mathrm{CN}$ & 24 & 96 \\
\hline 6 & D & $\mathrm{CH}_{3} \mathrm{CN}$ & 24 & 43 \\
\hline 7 & E1 & $\mathrm{CH}_{3} \mathrm{CN}$ & 24 & 94 \\
\hline 8 & E2 & $\mathrm{CH}_{3} \mathrm{CN}$ & 48 & 12 \\
\hline 9 & B1 & toluene & 24 & 99 \\
\hline 10 & B1 & DCM & 24 & 92 \\
\hline 11 & B1 & THF & 24 & 85 \\
\hline 12 & B1 & DCE & 24 & 52 \\
\hline 13 & B1 & 1,4-dioxane & 24 & 64 \\
\hline $14^{[c]}$ & B1 & $\mathrm{CH}_{3} \mathrm{CN}$ & 24 & 90 \\
\hline $15^{[\mathrm{d}]}$ & B1 & $\mathrm{CH}_{3} \mathrm{CN}$ & 24 & 37 \\
\hline $16^{[\mathrm{e}]}$ & B1 & $\mathrm{CH}_{3} \mathrm{CN}$ & 24 & 99 \\
\hline
\end{tabular}


[a] $\mathbf{1 a}(0.30 \mathrm{mmol}), \mathbf{2 a}(0.20 \mathrm{mmol}), \mathrm{NHC} \mathbf{A}(10 \mathrm{~mol} \%)$ or NHC precursor $(11 \mathrm{~mol} \%)$, DBU (10

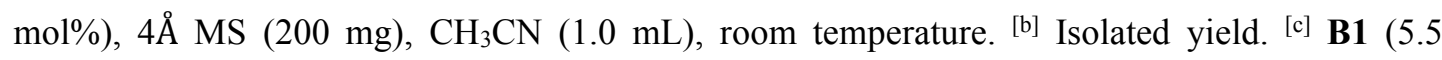
mol\%), DBU (5 mol\%). ${ }^{[\mathrm{d}]} \mathbf{1 a}(0.60 \mathrm{mmol}), \mathbf{2 a}(0.40 \mathrm{mmol}), \mathbf{B 1}(2.2 \mathrm{~mol} \%), \mathrm{DBU}(2 \mathrm{~mol} \%), 4 \AA$ MS (400 mg), $\mathrm{CH}_{3} \mathrm{CN}(1.0 \mathrm{~mL}) .{ }^{[\mathrm{e}]} \mathbf{1 a}(0.24 \mathrm{mmol}), \mathbf{2 a}(0.20 \mathrm{mmol}), \mathrm{NHC} \mathbf{B 1}(11 \mathrm{~mol} \%), \mathrm{DBU}$

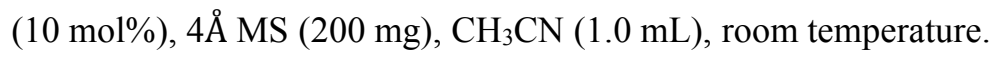

Scope of NHC-catalysed SuFEx reaction with phenols and alkyl alcohols. Having the optimal reaction conditions realized, we then evaluated the substrate scope and the results are summarized in Table 2. A variety of phenols underwent the SuFEx reaction with sulfonyl fluoride 1a smoothly to afford the corresponding sulfonate esters in high yields. The electronic properties and varied positions of the substituents on the aromatic ring of phenols had no obvious effect on the reaction yield (3b-3z). Many useful functional groups, such as halogen atoms (3b-3d, 3o-3q, 3t), nitrile (3e), aldehyde (3f), ketone (3g) and ester group (3h) were well tolerated. Pinacol boronic ester group substituted phenol coupled with sulfonyl fluoride 1a to produce $\mathbf{3 n}$ in $76 \%$ yield. Sterically hindered phenol $\mathbf{2 y}$ performed the reaction to give the desired product 3y in $95 \%$ yield. Both naphthols and tetrahydronaphthol were proved to be competent reactants for the click reaction, affording the corresponding products in high yield (3aa-3ac). Pyridinol underwent the reaction to produce 3ad in $95 \%$ yield. When 4-aminophenol was used for the reaction, the phenol hydroxy group selectively reacted with sulfonyl fluoride to give 3ae in $83 \%$ yield. Differently, hydroquinone showed no chemoselectivity for the reaction, and the corresponding disulfonylated 
product 3af were formed in $96 \%$ yield. The scope of sulfonyl fluorides was also examined. Both electron-withdrawing and -donating substituents substituted aryl sulfonyl fluorides reacted with phenol efficiently to furnish the corresponding sulfonates in high yields (3ag-3ar). The bulky aryl sulfonyl fluoride and heteroaryl derived sulfonyl fluorides reacted very well, giving the desired products efficiently (3as-3ay). Alkyl sulfonyl fluorides were proved to be good reactants for this SuFEx reaction, providing products $\mathbf{3 a z - 3} \mathbf{3 b}$ in high yields. The more challenging SuFEx click reaction of alcohols $\mathrm{s}^{32}$ and sulfonyl fluorides were also tested under the optimal reaction conditions. However, the reaction was sluggish and the product $\mathbf{3 b c}$ was only obtained in $21 \%$ yield (Table S2, entry 1, see Supporting Information). Pleasingly, switching the solvent from acetonitrile to dichloromethane and reversing the molar ratio of reactants, the yield of $3 \mathbf{b c}$ was improved to $84 \%$ (Table S2, entry 7). Under this modified reaction conditions, various alcohols underwent the click reaction to afford the corresponding sulfonates in high yields (3bd-3bn). Notably, different natural phenols and alcohols, such as estrone, $(+)-\delta$-tocopherol, deoxyarbutin, D-tyrosine and so on, underwent the reaction to afford the corresponding sulfonylated derivatives in high yields (3bo-3ca). This NHC-catalysed SuFEx click reaction can also be performed on a gram scale, and D-tyrosine derived sulfonate $\mathbf{3 b r}$ was obtained in $1.29 \mathrm{~g}$ and $96 \%$ yield.

Table 2 Substrates scope for the synthesis of sulfonates 


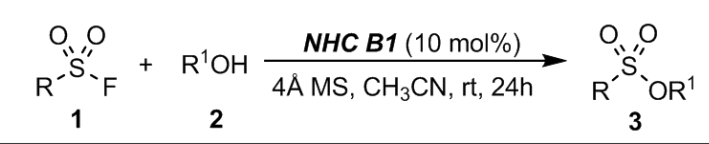

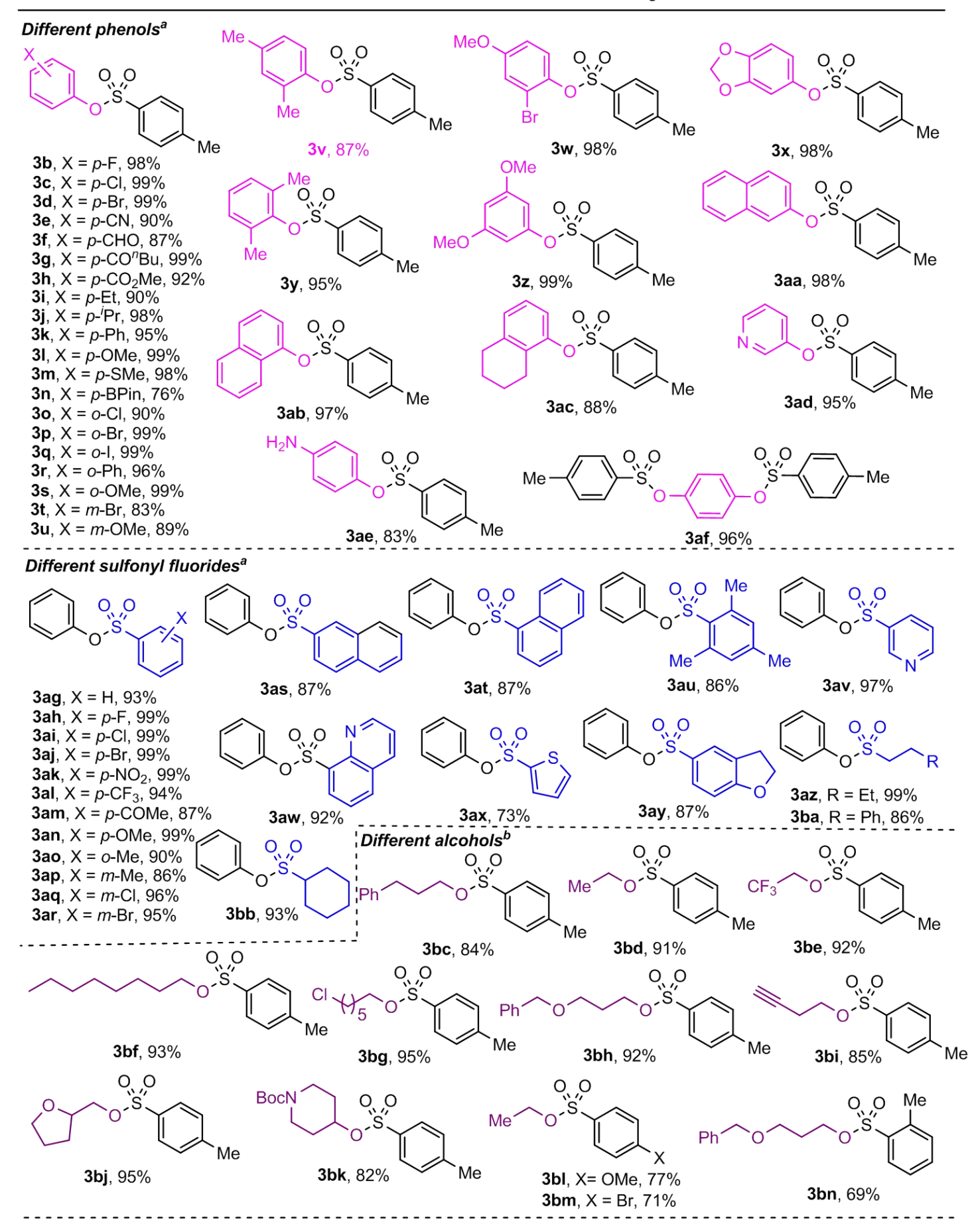




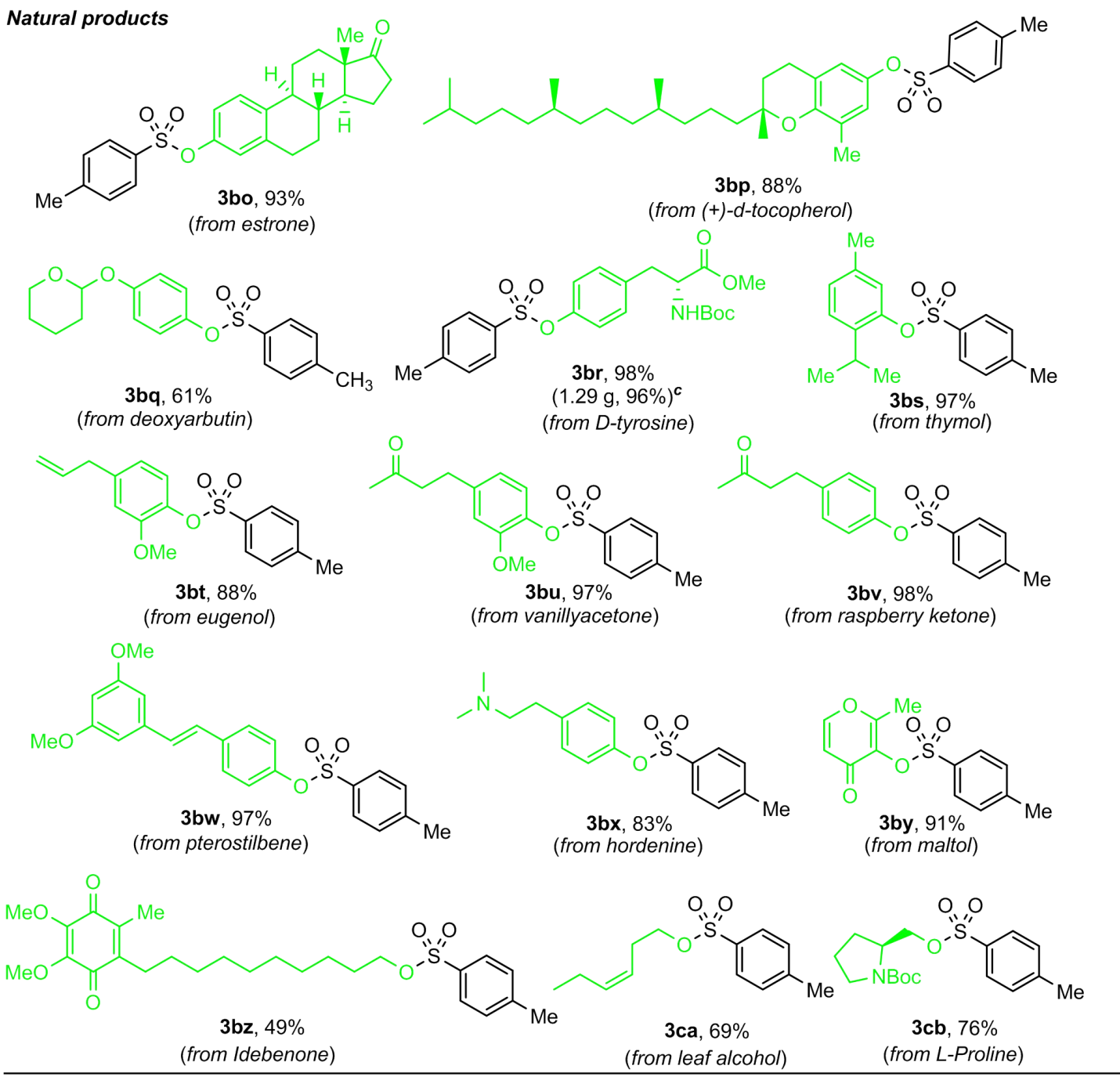

[a] 1 (0.24 mmol), 2 (0.20 mmol), NHC B1 (11 mol\%), DBU (10 mol\%), $4 \AA \mathrm{MS}(200 \mathrm{mg})$, $\mathrm{CH}_{3} \mathrm{CN}(1.0 \mathrm{~mL})$, room temperature, $24 \mathrm{~h}$, isolated yield. ${ }^{[b]} \mathbf{1}(0.20 \mathrm{mmol}), 2(0.40 \mathrm{mmol}), \mathrm{NHC}$ B1 (11 mol\%), DBU (10 mol\%), $4 \AA \mathrm{MS}(200 \mathrm{mg}), \mathrm{CH}_{2} \mathrm{Cl}_{2}(0.50 \mathrm{~mL})$, room temperature, $24 \mathrm{~h}$, isolated yield. ${ }^{[c]}$ 1a (3.6 mmol), methyl(tert-butoxycarbonyl)-D-tyrosinate (3.0 mmol), NHC B (5.5 mol\%), DBU (5 mol\%), $4 \AA \mathrm{MS}(3.0 \mathrm{~g}), \mathrm{CH}_{3} \mathrm{CN}(8 \mathrm{~mL})$, room temperature, $36 \mathrm{~h}$.

Scope of NHC-catalysed SuFEx reaction with amines. We next studied the SuFEx click reaction of sulfonyl fluorides and amines for the synthesis of sulfonamides. Under the standard reaction conditions, sulfonamide 5a was formed in very low yield (Table S3, entry 1, see Supporting Information). But fortunately, with the addition of 
$10 \mathrm{~mol} \% \mathrm{HOBt}$ as cocatalyst, the reaction yield of $\mathbf{5 a}$ was dramatically improved to 92\% (Table S3, entry 10). Further evaluation of the reaction media showed that quantitative yield was obtained when the reaction was conducted in toluene (Table S3, entry 28). Under this improved reaction conditions, a range of primary and secondary aliphatic amines were efficiently transformed into the corresponding sulfonamides in good to excellent yields (Table 3). Common functional groups, including halogen atoms (5b-5d), amine (5p), olefins (5q and 5ac) and heterocycles (5r and $\mathbf{5 s})$ can be incorporated into the amine substrates. Interestingly, diamine substrate $\mathbf{4 t}$ selectively underwent monosulfonation with sulfonyl fluoride to afford sulfonamide 5 t in $82 \%$ yield. Substrates derived from natural amino acids, such as $\mathbf{4 u}$ and $\mathbf{4 v}$, performed the SuFEx click reaction smoothly to afford the corresponding products in good yields. The natural leelamine $\mathbf{4} \mathbf{w}$ was proved to be a successful candidate for the reaction, giving sulfonamide $\mathbf{5 w}$ in $67 \%$ yield. Cyclic amines, such as pyrrolidine, piperidines, hexamethyleneimine, morpholines, and piperazines underwent the reaction to furnish the corresponding products in good to quantitative yields (5ad-5al). In addition, acid-sensitive groups such as Boc and ketal were well tolerated for the reaction (5ak and 5al). Increasing the amount of catalysts to $20 \mathrm{~mol} \%$, the less nucleophilic aromatic amines can also couple with sulfonyl fluorides to produce the desired sulfonamides in good to high yields (5am-5au). On the other hand, a range of aryl, heteroaryl and alkyl sulfonyl fluorides were proved to be very good electrophiles for the click reaction, delivering the desired products in moderate to excellent yields (5av-5bo). Notably, the reaction can be conducted on a gram scale and an excellent 
yield can be maintained (5ak).

Table 3 Scope of amines
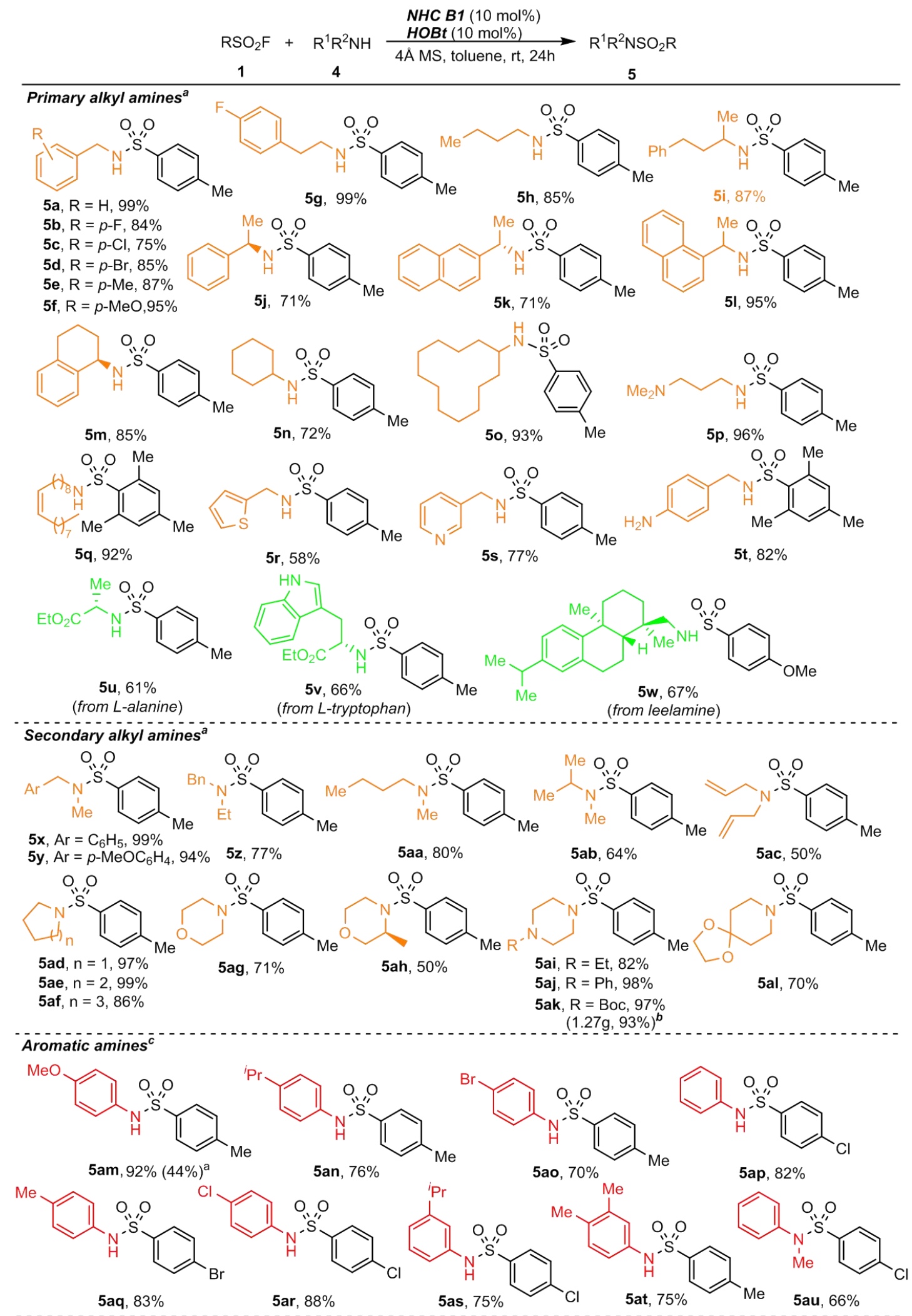


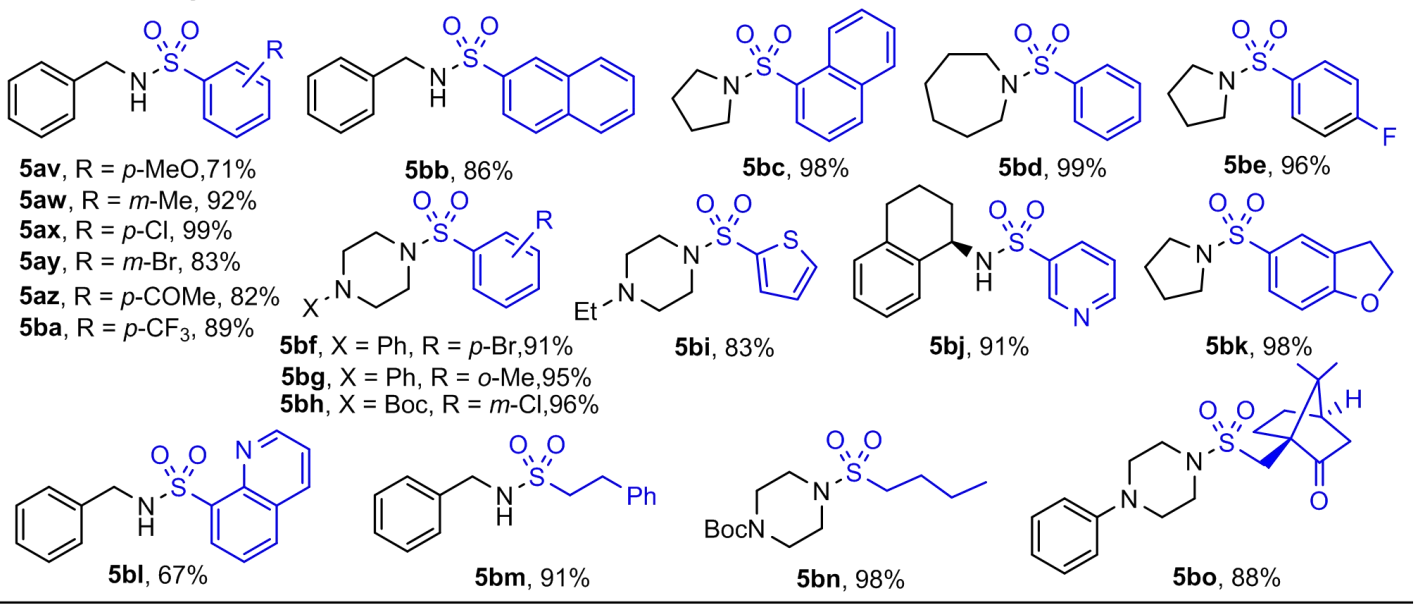

[a] 1 (0.30 mmol), 4 (0.20 mmol), NHC B1 (11 mol\%), DBU (10 mol\%), HOBt (10 mol\%), $4 \AA$

MS (200 mg), toluene $(1.0 \mathrm{~mL})$, room temperature, $24 \mathrm{~h}$, isolated yield. ${ }^{[b]} \mathbf{1 a}(6.0 \mathrm{mmol})$, N-Boc-piperazine (4.0 mmol), NHC B1 (2.2 mol\%), DBU (2 mol\%), HOBt (2 mol\%), 4A MS $(4.0 \mathrm{~g})$, toluene $(10 \mathrm{~mL})$, room temperature, $48 \mathrm{~h} .{ }^{[\mathrm{c}]} \mathbf{1}(0.30 \mathrm{mmol}), 4(0.20 \mathrm{mmol})$, NHC B1 $(22$ mol\%), DBU (20 mol\%), HOBt (20 mol\%), 4A MS (200 mg), toluene $(1.0 \mathrm{~mL})$, room temperature, $24 \mathrm{~h}$, isolated yield.

Mechanistic studies. Based on the previous studies of the Brønsted basicity of $\mathrm{NHCs}^{58-61}$ and the NMR experimental results (see Supporting Information), a plausible mechanism for the reaction was proposed and depicted in Scheme 1. NHC acts as a carbon-centered Brønsted base to attack the acidic proton of phenols or alcohols through hydrogen bonding to form an oxy anion/azolium ion complex I, which might trigger the following SuFEx click reaction with sulfonyl fluoride to produce sulfonate product with releasing of free carbene. For NHC-catalysed synthesis of sulfonamide, NHC firstly interacts with HOBt to form intermediate II, which reacts with sulfonyl fluoride to form sulfonate III. The active sulfonated III 
subsequently undergoes ester-aminolysis reaction ${ }^{35}$ with an amine to produce the sulfonamide product with releasing of HOBt.

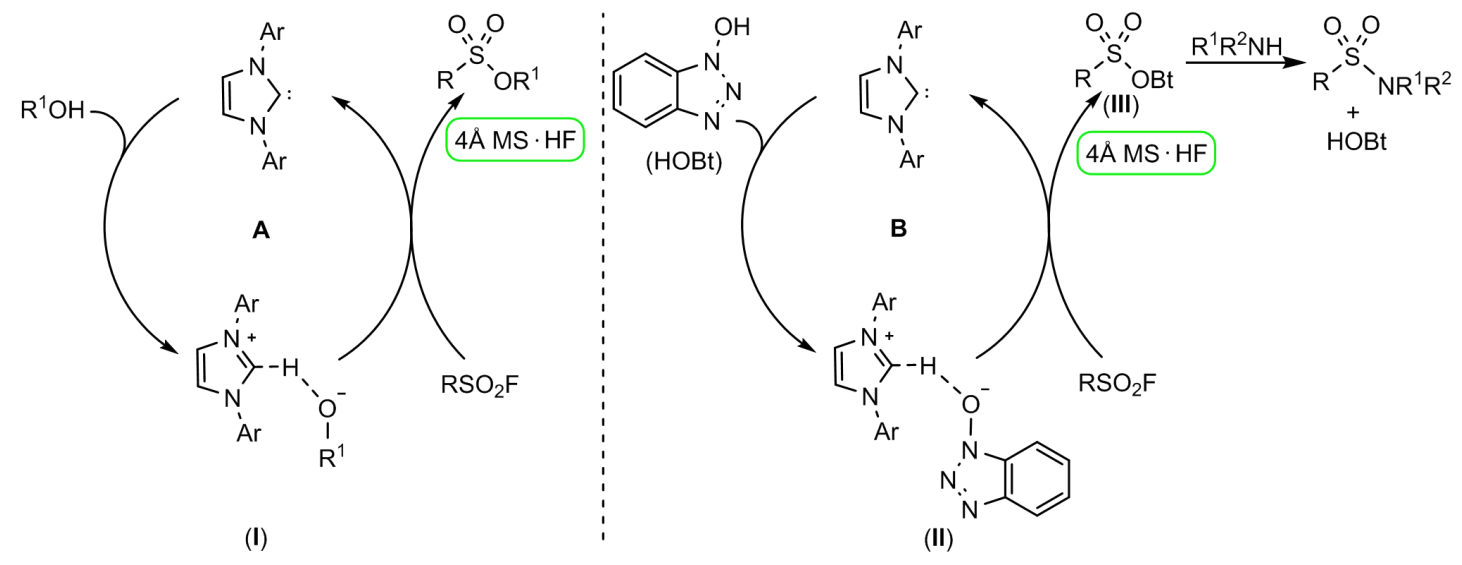

Scheme 1 Proposed Mechanism

\section{Conclusion}

In summary, NHC-catalysed SuFEx click reactions of sulfonyl fluorides have been demonstrated. The silicon and base-free conditions, broad substrate scope, generally high yields and easy scalability provide a novel organocatalytic protocol for the synthesis of sulfonates and sulfonamides. Further applications of NHCs in other SuFEx click reactions are ongoing in our laboratory.

\section{Methods}

General procedure for the synthesis of sulfonic ester 3.

To a suspension of imidazolium B1 $(0.022 \mathrm{mmol}, 7.5 \mathrm{mg}, 11 \mathrm{~mol} \%)$ and $4 \AA \mathrm{MS}(200 \mathrm{mg})$ in anhydrous $\mathrm{CH}_{3} \mathrm{CN}$ $(1.0 \mathrm{~mL})$ was added DBU $(0.02 \mathrm{mmol}, 3.0 \mathrm{mg}, 10 \mathrm{~mol} \%)$ under a nitrogen atmosphere. The mixture was stirred at ambient temperature for $0.5 \mathrm{~h}$. Sulfonyl fluoride 1 ( $0.24 \mathrm{mmol}, 1.2$ equiv) and phenol 2 ( $0.20 \mathrm{mmol}, 1.0$ equiv) were added subsequently and the solution was stirred at ambient temperature until full consumption of the starting phenol indicated by TLC. The reaction mixture was then diluted with ethyl acetate, filtered through a short pad of 
silica gel and concentrated. The crude product was purified by flash column chromatography on silica gel to afford the pure product.

\section{Data availability}

Experimental procedures, mechanism studies and characterization of the compounds are available in the Supplementary Information. All other data are available from the corresponding authors upon reasonable request.

\section{References}

1. Spillane, W. \& Malaubier, J. B. Sulfamic acid and its N-and O-substituted derivatives. Chem. Rev. 114, 2507-2586 (2014).

2. Zhao, C., Rakesh, K. P., Ravidar, L., Fang, W.-Y. \& Qin, H.-L. Pharmaceutical andmedicinal significance of sulfur ( $\mathrm{S}^{\mathrm{VI}}$ )-Containing motifs for drug discovery: a critical review. Eur. J. Med. Chem. 162, 679-734 (2019).

3. Scott, K. A. \& Njardarson, J. T. Analysis of US FDA-approved drugs containing sulfur atoms. Top. Curr. Chem. 376, 5 (2018).

4. Apaydın, S. \& Török, M. Sulfonamide derivatives as multi-target agents for complex diseases. Bioorg. Med. Chem. Lett. 29, 2042-2050 (2019).

5. Winum, J. Y., Scozzafava, A., Montero, J. L. \& Supuran, C. T. Sulfamates and their therapeutic potential. Med. Res. Rev. 25, 186-228 (2005).

6. Winum, J. Y., Scozzafava, A., Montero, J. L. \& Supuran, C. T. Therapeutic application of sulfamates. Expert. Opin. Ther. Pat. 14, 1273-1308 (2005).

7. Kim, T., McCarver, S. J., Lee, C. \& MacMillan, D. W. C. Sulfonamidation of aryl and heteroaryl halides through photo-sensitized nickel catalysis. Angew. Chem., Int. Ed. 57, 3488-3492 (2018).

8. Chen, Y.-D., Murray, P. R. D., Davies, A. T. \& Willis, M. C. Direct copper-catalyzed three-component synthesis of sulfonamides. J. Am. Chem. Soc. 140, 8781-8787 (2018). 
9. Zhao, X.-H., Xu, H.-Y., Huang, X.-L. \& Zhou, J.-S. Asymmetric stepwise reductive amination of sulfonamides, sulfamates, and a phosphinamide by nickel catalysis. Angew. Chem. Int. Ed. 58, 292-296 (2019).

10. Blum, S. P., Karakaya, T., Schollmeyer, D., Klapars, A. \& Waldvogel, S. R. metal-free electrochemical synthesis of sulfonamides directly from(hetero)arenes, $\mathrm{SO}_{2}$, and amines Angew. Chem. Int. Ed. 60, 5056-5062 (2021).

11. Aliyu, M. A. \& Lee, S. One-pot synthesis of pentafluorophenyl sulfonic esters via copper-catalyzed reaction of aryl diazonium salts, DABSO, and pentafluorophenol. Org. Lett. 23, 4516-4520 (2021).

12. Bogolubsky, A. V. et al. Sulfonyl fluorides as alternative to sulfonyl chlorides in parallel synthesis of aliphatic sulfonamides. ACS. Comb. Sci. 16, 192-197 (2014).

13. a) Dong, J.-J., Krasnova, L., Finn, M. G. \& Sharpless, K. B. Sulfur(VI) fluoride exchange (SuFEx): another good reaction for click chemistry. Angew. Chem. Int. Ed. 53, 9430-9448 (2014).

14. Barrow, A. S. et al. The growing applications of SuFEx click chemistry. Chem. Soc. Rev. 48, 4731-4758 (2019).

15. Lee, C. et al. The emerging applications of sulfur(VI) fluorides in catalysis. ACS Catal. 11, 6578-6589 (2021).

16. Meng, G.-Y. et al. Modular click chemistry libraries for functional screens using a diazotizing reagent. Nature 574, 86-89 (2019).

17. Liu, C. et al. A general approach to O-sulfation by a sulfur(VI) fluoride exchange reaction. Angew. Chem. Int. Ed. 59, 18435-18441 (2020).

18. Gao, B., Li, S.-H., Wu, P., Moses, J. E. \& Sharpless, K. B. SuFEx chemistry of thionyl tetrafluoride $\left(\mathrm{SOF}_{4}\right)$ with organolithium nucleophiles: synthesis of sulfonimidoyl fluorides, sulfoximines, sulfonimidamides, and sulfonimidates. Angew. Chem. Int. Ed. 57, 1939-1943 (2018).

19. Guo, T.-J. et al. A new portal to SuFEx click chemistry: a stable fluorosulfuryl imidazolium salt emerging as 
an " $\mathrm{F}^{-} \mathrm{SO}_{2}{ }^{+}$" donor of unprecedented reactivity, selectivity, and scope. Angew. Chem. Int. Ed. 57, 2605-2610 (2018).

20. Hmissa, T. et al. Autocatalytic synthesis of bifluoride ionic liquids by SuFEx click chemistry. Angew. Chem. Int. Ed. 57, 16005-16009 (2018).

21. Liang, D.-D. et al. Silicon-free SuFEx reactions of sulfonimidoyl fluorides: scope, enantioselectivity, and mechanism. Angew. Chem. Int. Ed. 59, 7494-7500 (2020).

22. Liu, J. et al. A Genetically encoded fluorosulfonyloxybenzoyl-1-lysine for expansive covalent bonding of proteins via SuFEx chemistry. J. Am. Chem. Soc. 143, 10341-10351 (2021).

23. Liu, F. et al. Biocompatible SuFEx click chemistry: thionyl tetrafluoride $\left(\mathrm{SOF}_{4}\right)$-derived connective hubs for bioconjugation to DNA and proteins. Angew. Chem. Int. Ed. 58. 8029-8033 (2019).

24. Yang, B. et al. Genetically introducing biochemically reactive amino acids dehydroalanine and dehydrobutyrine in proteins. J. Am. Chem. Soc. 141, 7698-7703 (2019).

25. Chen, W.-T. et al. Arylfluorosulfates inactivate intracellular lipid binding protein(s) through chemoselective SuFEx reaction with a binding site tyr residue. J. Am. Chem. Soc. 138, 7353-7364 (2016).

26. Liu, Z.-L. et al. SuFEx click chemistry enabled late-stage drug functionalization. J. Am. Chem. Soc. 140, 2919-2925 (2018).

27. Smedley, C. J. et al. Diversity oriented clicking (DOC): divergent synthesis of SuFExable pharmacophores from 2-substituted-alkynyl-1-sulfonyl fluoride (SASF) hubs. Angew. Chem. Int. Ed. 59, 12460-12469 (2020).

28. Kitamura, S. et al. Sulfur(VI) fluoride exchange (SuFEx)-enabled high-throughput medicinal chemistry. J. Am.

Chem. Soc. 142, 10899-10904 (2020).

29. Li, S.-H. et al. SuFExable polymers with helical structures derived from thionyl tetrafluoride Nat. Chem. 13, 858-867 (2021). 
30. Gao, B. et al. Bifluoride-catalysed sulfur(VI) fluoride exchange reaction for the synthesis of polysulfates and polysulfonates. Nat. Chem. 9, 1083-1088 (2017).

31. Wang, H. et al. SuFEx-based polysulfonate formation from ethenesulfonyl fluoride-amine adducts. Angew. Chem. Int. Ed. 56, 11203-11208 (2017).

32. Smedley, C. J. et al. Accelerated SuFEx click chemistry for modular synthesis. Angew. Chem. Int. Ed. doi: 10.1002/anie.202112375 (2021).

33. Mukherjee, P. et al. Sulfonamide synthesis via calcium triflimide activation of sulfonyl fluorides, Org. Lett. 20, 3943-3947 (2018).

34. Wei, M.-J. et al. A broad-spectrum catalytic amidation of sulfonyl fluorides and fluorosulfates, Angew. Chem. Int. Ed. 60, 7397-7404 (2021).

35. Hopkinson, M. N., Richter, C., Schedler, M. \& Glorius, F. An overview of N-heterocyclic carbenes. Nature 510, 485-496 (2014).

36. Flanigan, D. M., Romanov-Michailidis, F., White, N. A. \& Rovis, T. Organocatalytic reactions enabled by N-heterocyclic carbenes. Chem. Rev. 115, 9307-9387 (2015).

37. Bellotti, P., Koy, M., Hopkinson, M. N. \& Glorius, F., Recent advances in the chemistry and applications of N-heterocyclic carbenes. Nat. Rev. Chem. 5, 711-725 (2021).

38. Peng, Q.-P., Guo, D.-H., Bie, J.-B. \& Wang, J. Catalytic enantioselective aza-benzoin reactions of aldehydes with 2H-azirines. Angew. Chem. Int. Ed. 57, 3767-3771 (2018).

39. Zhang, G.-X. et al. Dynamic kinetic resolution enabled by intramolecular benzoin reaction: synthetic applications and mechanistic insights. J. Am. Chem. Soc. 138, 7932-7938 (2016).

40. Liu, Q., Perreault, S. \& Rovis, T. Catalytic asymmetric intermolecular stetter reaction of glyoxamides with alkylidenemalonates. J. Am. Chem. Soc. 130, 14066-14067 (2008). 
41. Fernando, J. E. M., Nakano, Y., Zhang, C.-H. \& Lupton, D. W. Enantioselective N-heterocyclic carbene catalysis that exploits imine umpolung. Angew. Chem. Int. Ed. 58, 4007-4011 (2019).

42. White, N. A. \& Rovis, T. Enantioselective N-Heterocyclic carbene-catalyzed $\beta$-hydroxylation of enals using nitroarenes: an atom transfer reaction that proceeds via single electron transfer. J. Am. Chem. Soc. 136, 14674-14677 (2014).

43. Guo, C., Sahoo B., Daniliuc, C. G. \& Glorius, F. N-heterocyclic carbene catalyzed switchable reactions of enals with azoalkenes: formal [4+3] and [4+1] annulations for the synthesis of 1,2-diazepines and pyrazoles. J. Am. Chem. Soc. 136, 17402-17405 (2014).

44. Chen, X.-Y., Liu, Q., Chauhan, P. \& Enders, D. N-heterocyclic carbene catalysis via azolium dienolates: an efficient strategy for remote enantioselective functionalizations. Angew. Chem. Int. Ed. 57, 3862-3873 (2018).

45. Zhang, C.-L., Gao, Y.-Y., Wang, H.-Y., Zhou, B.-A. \& Ye, S. Enantioselective Synthesis of Axially Chiral Benzothiophene/Benzofuran-Fused Biaryls by N-Heterocyclic Carbene Catalyzed Arene Formation, Angew. Chem. Int. Ed. 60, 13918-13922 (2021).

46. Vora, H. U. \& Rovis, T. Nucleophilic carbene and HOAt relay catalysis in an amide bond coupling: an orthogonal peptide bond forming reaction. J. Am. Chem. Soc. 129, 13796-13797 (2007).

47. Yatham, V. R. et al. 1,4-Bis-Dipp/Mes-1,2,4-Triazolylidenes: Carbene Catalysts That Efficiently Overcome Steric Hindrance in the Redox Esterification of $\alpha$ - and $\beta$-Substituted $\alpha, \beta$-Enals, J. Am. Chem. Soc. 138, 2670-2677 (2016).

48. Liu, K. \& Studer, A. Direct $\alpha$-acylation of alkenes via N-heterocyclic carbene, sulfinate, and photoredox cooperative triple catalysis. J. Am. Chem. Soc. 143, 4903-4909 (2021).

49. Bay, A. V. et al. Light-driven carbene catalysis for the synthesis of aliphatic and $\alpha$-amino ketones. Angew.

Chem. Int. Ed. 60, 17925-17931 (2021). 
50. Dai, L., Xia, Z.-H., Gao, Y.-Y., Gao, Z.-H. \& Ye, S. Visible-light-driven N-heterocyclic carbene catalyzed $\gamma$ and €-alkylation with alkyl radicals. Angew. Chem. Int. Ed. 58, 18124-18130 (2019).

51. Geng, H. et al. Practical synthesis of $\mathrm{Cl}$ deuterated aldehydes enabled by NHC catalysis, Nat. Catal. 2, 1071-1077 (2019).

52. Singha, S., Serrano, E., Mondal , S., Daniliuc, C. G. \& Glorius, F. Diastereodivergent synthesis of enantioenriche $\alpha, \beta$-disubstituted $\gamma$-butyrolactones via cooperative $\mathrm{N}$-heterocyclic carbene and Ir catalysis, Nat.

Catal. 3, 48-54 (2020).

53. Levens, A. A., Candish, L. \& Lupton, D. W. N-heterocyclic carbene catalyzed synthesis of $\delta$-sultones via $\alpha$, $\beta$-unsaturated sulfonyl azolium intermediates. Angew. Chem. Int. Ed. 54, 11780-11784 (2015).

54. Yang, X. et al. Carbene-catalyzed activation of remote nitrogen atoms of (benz)imidazole-derived aldimines for enantioselective synthesis of heterocycles. Angew. Chem. Int. Ed. 60, 7906-7912 (2021).

55 Grasa, G. A., Kissling, R. M. \& Nolan, S. P. N-heterocyclic carbenes as versatile nucleophilic catalysts for transesterification/acylation reactions. Org. Lett. 4, 3583-3586 (2002);

56. Nyce, G. W., Lamboy, J. A., Connor, E. F., Waymouth, R. M. \& Hedrick, J. L. Expanding the catalytic activity of nucleophilic N-heterocyclic carbenes for transesterification reactions. Org. Lett. 4, 3587-3590 (2002).

57. Phillips, E. M., Riedrich, M. \& Scheidt, K. A. N-heterocyclic carbene-catalyzed conjugate additions of alcohols. J. Am. Chem. Soc. 132, 13179-13181 (2010).

58. Chen, J.-A. \& Huang, Y. Asymmetric catalysis with N-heterocyclic carbenes as non-covalent chiral templates. Nat. Commun. 5, 3437 (2014).

59. Wang, L., Chen, J. \& Huang, Y. Highly enantioselective aza-michael reaction between alkyl amines and $\beta$-trifluoromethyl $\beta$-aryl nitroolefins. Angew. Chem. Int. Ed. 54, 15414-15418 (2015). 
60. Cong, Z.-S. et al. N-Heterocyclic carbene-catalyzed sulfa-Michael addition of enals. Chem. Commun. 53,

13129-13132. (2017).

\section{Acknowledgements}

Financial support from the National Natural Science Foundation of China (grant numbers 21662029 and 21262027

for G.D.) is acknowledged.

\section{Author contributions}

M.L., J.L., Y.X. and Z.C.conducted and analysed the experiments. G.D., B.D. and L.H. designed and directed the project and G.D. and L.H. wrote the manuscript.

\section{Competing interests}

The authors declare no competing interests. 


\section{Graphical abstract}

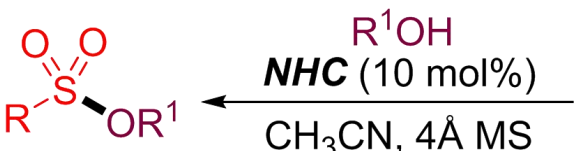

80 examples rt, $24 \mathrm{~h}$

up to $99 \%$ yield

$\mathrm{R}, \mathrm{R}^{1}=$ (hetero)aryl, alkyl

$\mathrm{R}^{2}, \mathrm{R}^{3}=$ (hetero)aryl, alkyl, $\mathrm{H}$

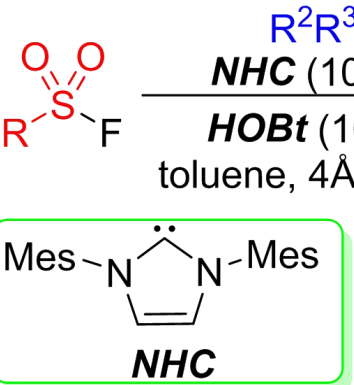

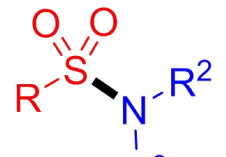

$\mathrm{R}^{3}$

67 examples

up to $99 \%$ yield
- silicon and base free
broad substrate scope
- high reaction yield
- mild conditions
gram-scale synthesis
17 natural products 